\title{
Swelling of Poly(methyl acrylate) Brushes in Acetone Vapor
}

\author{
Rens J. Horst, ${ }^{\perp}$ Maria Brió Pérez, ${ }^{\perp}$ Rick Cohen, Marco Cirelli, Paloma S. Dueñas Robles, \\ Maria G. Elshof, Aleksandar Andreski, Mark A. Hempenius, Nieck E. Benes, Cas Damen, \\ and Sissi de Beer*
}

Cite This: Langmuir 2020, 36, 12053-12060

Read Online

ACCESS | Lلll Metrics \& More | 回 Article Recommendations | st Supporting Information

ABSTRACT: Sensor platforms can benefit from the incorporation of polymer brushes since brushes can concentrate the analyte near the sensor surface. Brushes that absorb acetone vapor are of particular interest since acetone is an important marker for biological processes. We present a simple procedure to synthesize acetoneresponsive poly(methyl acrylate) brushes. Using spectroscopic ellipsometry, we show that these brushes respond within seconds and swell by more than $30 \%$ when exposed

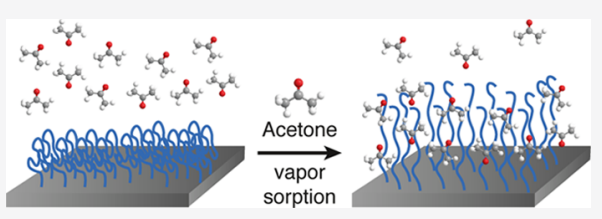
to acetone vapor. Moreover, quartz crystal microbalance measurements demonstrate that the brushes can be exploited to increase the acetone detection sensitivity of sensors by more than a factor 6. Surprisingly, we find that the swelling ratio of the brushes in acetone vapor is independent of the grafting density and the degree of polymerization of the polymers in the brush. This is qualitatively different from swelling of the same brushes in liquid environments, where the swelling ratio decreases for increasing grafting densities. Yet, it indicates that the brushes are robust and reproducible candidates for implementation in vapor sensor systems.

\section{INTRODUCTION}

Continuous monitoring of scents is an excellent noninvasive technique to track the health state of patients, crops in warehouses, or cattle in stables. Therefore, in recent years, much effort has been devoted to developing sensors that can track scents. ${ }^{1-4}$ Such so-called electronic noses have to be able to detect trace amounts of a particular fragrance that consists of a predefined mixture of volatile organic compounds (VOCs). Inspired by the (human) olfactory system, ${ }^{5}$ odor identification can be achieved by arrays of sensors that bear different surface functionalizations. ${ }^{6}$ These coatings will bind vapor molecules selectively in distinct ratios by affinity, without the necessity for specificity. For each fragrance, the array will constitute a fingerprint that is recognized by the electronic nose. $^{7}$ The sensitivity of the sensor depends on the amount of VOC molecules that can be bound to the surface at low VOC concentrations. Therefore, polymer brushes could aid as selective absorbers to enhance this.

Polymer brushes are surface functionalizations that are composed of long macromolecules that are attached to a substrate by one end at a sufficiently high density such that they stretch away from the surface. ${ }^{8}$ When these brushes are exposed to a liquid or a vapor that is a good solvent for the polymers, they will swell and absorb the fluid. This effect has been employed to design effective lubricants ${ }^{9-11}$ and nonfouling surfaces. ${ }^{12}$ Moreover, advanced brush systems have been designed to aid sensing platforms by reducing nonspecific adsorption and binding large amounts of analyte molecules in liquids $^{13-15}$ and in gaseous ${ }^{16}$ environments. Because of the latter capacity, we anticipate that brushes are promising surface functionalizations to increase the sensitivity of electronic noses too.

Acetone is an important marker in the detection of food degradation or for monitoring diabetes. ${ }^{17}$ Therefore, brushes that swell in acetone will be a critical component of electronic noses. In this article, we present a simple synthesis route to prepare brushes that strongly and quickly swell in acetone vapor. The brushes are composed of poly(methyl acrylate) (PMA) for which we designed a synthesis scheme using the facile and inexpensive surface-initiated activators regenerated by the electron transfer atom transfer radical polymerization (SI-ARGET-ATRP) method. ${ }^{18,19}$ PMA was selected since acetone is a good solvent for the polymer, with reported swelling ratios in liquid up to $\sim 4 .^{20}$ We show that the brushes swell by more than $30 \%$ when they are brought in contact with nearly saturated acetone vapor. Moreover, quartz crystal microbalance (QCM) measurements demonstrate that we can improve the sensitivity of mass-based, gravimetric vapor sensors by more than a factor 6 compared to surfaces without brush coatings.

Received: August 24, 2020

Published: September 30, 2020 


\section{Scheme 1. Synthesis Scheme for Grafting PMA Brushes from $\mathrm{SiO}_{2}$ Using Surface-Initiated ARGET-ATRP}

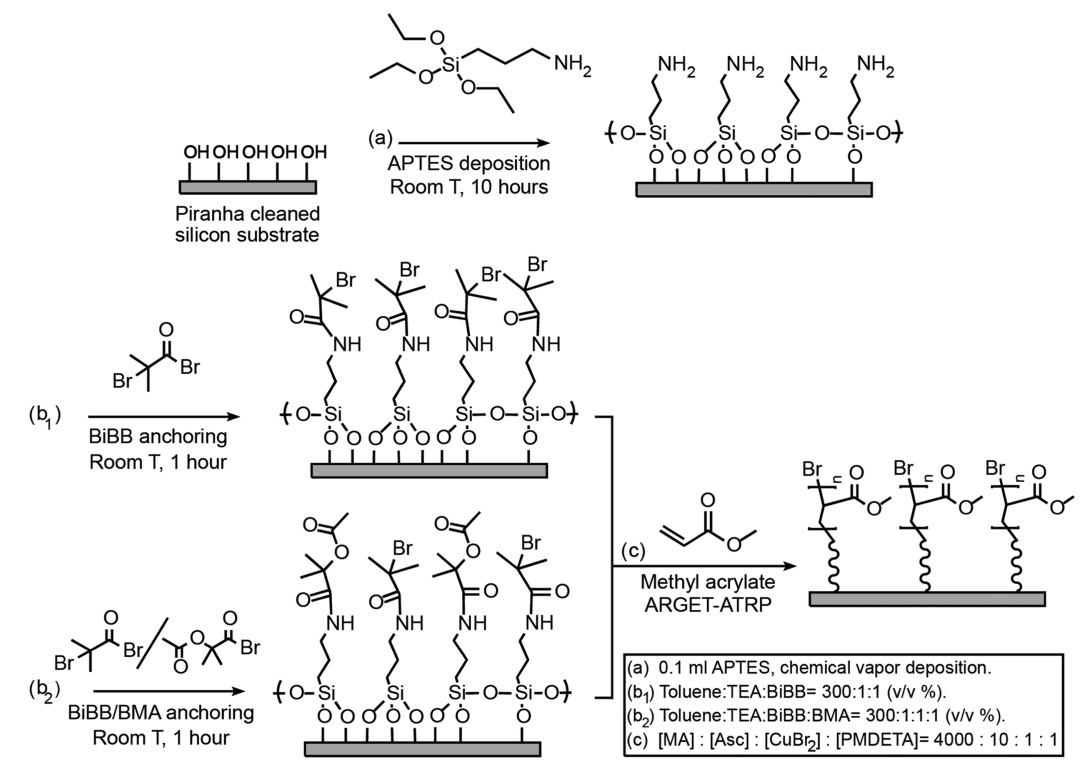

\section{EXPERIMENTAL SECTION}

Materials. Silicon wafers $(100.0 \pm 0.5 \mathrm{~mm}$ diameter and $525 \pm 25$ $\mu \mathrm{m}$ thickness, boron-doped with (100) orientation, 5-10 $\Omega \mathrm{cm}$, Okmetic) were cut in $1 \times 1 \mathrm{~cm}^{2}$ for atomic force microscopy (AFM) studies, in $5 \times 2 \mathrm{~cm}^{2}$ for ellipsometry measurements and were used as surface for polymer brush functionalizations. Silicon dioxide-coated QCM plates (AT cut, $10 \mathrm{MHz}, \mathrm{SiO}_{2}$ QSensor QSX $303 \mathrm{SiO}_{2}$, Qsense) were also used as surfaces for polymer brush functionalizations. Prior to use, the QCM plates were cleaned following QSense cleaning protocols. Methyl acrylate (MA, 99\%, CAS 96-33-3), copper(II) bromide $\left(\mathrm{CuBr}_{2}, 99 \%\right.$, CAS 7788-45-9), $\alpha$-bromoisobutyryl bromide (BiBB, 98\%, CAS 20769-85-1), ethyl $\alpha$-bromoisobutyrate (EBiBB, 98\%, CAS 600-00-0), $N, N, N^{\prime}, N^{\prime \prime}, N^{\prime \prime}$-pentamethyldiethylenetriamine (PMDETA, 99\%, CAS 3030-47-5), triethylamine (TEA, 99\%, CAS 121-44-8), (3-aminopropyl) triethoxysilane (APTES, 99\%, CAS 919-30-2), ascorbic acid (>99\%, CAS 50-81-7), 1-bromocarbonyl-1-methylethyl acetate (BMA, 96\%, CAS 400635-674), sulfuric acid $\left(\mathrm{H}_{2} \mathrm{SO}_{4}, 99 \%\right.$, CAS 7664-93-4), dimethyl sulfoxide (DMSO, 99\%, CAS 67-68-5), toluene (99\%, CAS 108-88-3), chloroform- $d_{6}$ (CF-d, 99,8\%, CAS 865-49-6), and hydrogen peroxide $\left(\mathrm{H}_{2} \mathrm{O}_{2}, 30 \%\right.$, CAS 7722-84-1) were purchased from Sigma-Aldrich and used as received without purification. Milli- $Q$ water was made from a Milli-Q Advange A 10 purification system (Millipore, Billerica, $\mathrm{Ma})$. A Figaro TGS $2620 \mathrm{C}$ gas sensor was used for acetone detection.

Methods. Synthesis of PMA Brushes. First, the substrates were thoroughly cleaned with piranha solution $\left(\mathrm{H}_{2} \mathrm{SO}_{4} / \mathrm{H}_{2} \mathrm{O}_{2}=3: 1 \mathrm{v} / \mathrm{v}\right.$ $\%)$, subsequently rinsed with ethanol and water, and dried under a nitrogen stream. Next, APTES layers were used as anchoring points and deposited onto the clean substrates by means of chemical vapor deposition. The process was conducted in a dessicator, by storing together the substrates and $0.1 \mathrm{~mL}$ of APTES solution. Vacuum was first applied for $15 \mathrm{~min}$, and the vapor deposition was allowed to proceed overnight. The samples were again rinsed with water and ethanol and dried under a nitrogen stream. When grafting APTES molecules on substrates, different binding possibilities may occur, such as the silanol $(\mathrm{Si}-\mathrm{O})$ group binding between neighboring silane molecules in both horizontal and/or vertical direction and selfpolymerization reactions. Moreover, structural irregularities are also a common phenomenon, such as hydrogen bonding or electrostatically attached silane molecules. ${ }^{21,22}$ A simplified schematic on the binding of APTES is shown in Scheme 1.

Consecutively, $\mathrm{BiBB}$ initiator molecules were bound to the anchoring layers. The substrates were placed in a sample holder to avoid sample damaging and salt adhesion on the substrates during the reaction. The sample holder with the substrates in it was placed inside a beaker containing a solution of cold toluene and triethylamine (TEA), followed by a dropwise addition of the initiator. The ratios between the used solutions were toluene/TEA/BiBB $=300: 1: 1(\mathrm{v} / \mathrm{v}$ $\%)$. The reaction was carried out for $1 \mathrm{~h}$. Next, the substrates were rinsed with ethanol and water and dried under a nitrogen stream.

Next, PMA brushes were synthesized via surface-initiated activators regenerated by electron transfer atom transfer radical polymerization (SI-ARGET-ATRP), one of the newest controlled radical polymerization procedures that uses much lower concentrations of catalysts compared to conventional ATRP methods. ${ }^{23}$ This kind of polymerization makes the future scaling up more feasible and produces polymer brushes with higher purities. ${ }^{24}$ In a round-bottom flask, MA $(10 \mathrm{~mL}, 110.3 \mathrm{mmol})$ and PMDETA $(5.6 \mu \mathrm{L}, 0.027 \mathrm{mmol})$ were dissolved under constant stirring in dimethylformamide (DMF) (10 $\mathrm{mL}$ ). The mixture was sealed with a septum and purged with nitrogen for at least $1 \mathrm{~min} / \mathrm{mL}$ of solution. In a second round-bottom flask, $\mathrm{CuBr}_{2}(6.1 \mathrm{mg}, 0.027 \mathrm{mmol})$ and ascorbic acid $(48.6 \mathrm{mg}, 0.27 \mathrm{mmol})$ powders were also purged with nitrogen flows for at least $30 \mathrm{~min}$. The reactant molar ratio was $[\mathrm{MA}] /[\mathrm{Asc}] /\left[\mathrm{CuBr}_{2}\right] /[\mathrm{PMDETA}]=$ 4000:10:1:1. Consecutively, the monomer solution was transferred with a purged syringe to the flask containing the powders and was bubbled with nitrogen and thoroughly stirred until complete powder dissolution. After that, the mixture containing monomer, ligand, reducing agent, and catalyst was transferred to the flask containing the substrates $\left(5 \times 2 \mathrm{~cm}^{2}\right.$ wafers for ellipsometry measurements and $1 \times 1$ $\mathrm{cm}^{2}$ for AFM measurements). The surface-initiated polymerization was left to proceed for several reaction times: 1, 2, 3, 4, and $18 \mathrm{~h}$. By removing the septum which sealed the flask and allowing oxygen inside the container, the reaction was stopped. Finally, the substrates were thoroughly rinsed with water, ethanol, and acetone and flushed with nitrogen.

Grafting Density Variations. Polymer brushes with various grafting densities were grown for the study of its influence in the swelling responses of the brushes in acetone vapors. Piranha cleaned substrates were first functionalized with APTES anchors following the previously described methods. Next, a mixture of reactive BiBB initiators and nonreactive 1-bromocarbonyl-1-methylethyl acetate (BMA) was grafted on the substrates. Four different solutions were used: 100 vol \% BiBB, 50 vol \% BiBB, 25 vol \% BiBB, and 12.5 vol \% BiBB (with a toluene/TEA/BiBBV+VBMA ratio of 300:1:1 (v/v \%)). The BiBB and BMA parts were premixed in toluene and added to the beaker containing the substrates, followed by a dropwise addition of TEA. The reactions were allowed to take place for $1 \mathrm{~h}$, and the substrates were rinsed with water and ethanol afterward. Next, polymer brushes were grown from the functionalized substrates, obtaining different 
grafting densities due to the variations of the $[\mathrm{BiBB}] /[\mathrm{BMA}]$ ratios. The polymer brush synthesis procedure is the same as previously described. The change in the relative surface coverage by the change in the concentration of BMA molecules was studied by means of Xray photoelectron spectroscopy (XPS).

Polymerization in Solution. In a round-bottom flask, MA $(10 \mathrm{~mL}$, $110.3 \mathrm{mmol})$ and PMDETA $(5.76 \mu \mathrm{L}, 0.027 \mathrm{mmol})$ were dissolved under constant stirring in DMF $(9 \mathrm{~mL})$. The mixture was sealed with a septum and purged with nitrogen for at least $1 \mathrm{~min} / \mathrm{mL}$ of solution. In a second round-bottom flask, $\mathrm{CuBr}_{2}(6.1 \mathrm{mg}, 0.027 \mathrm{mmol})$ and ascorbic acid $(48.6 \mathrm{mg}, 0.27 \mathrm{mmol}$ ) were also purged with nitrogen flows for at least $30 \mathrm{~min}$. In a glass vial, the sacrificial initiator, EBiBB $(2 \mu \mathrm{L}, 0.0138 \mathrm{mmol})$, was dissolved in $1 \mathrm{~mL}$ of DMF. The selected internal standard for proton nuclear magnetic resonance ( $\left.{ }^{1} \mathrm{H}-\mathrm{NMR}\right)$ measurements (dimethyl sulfoxide (DMSO), $0.1 \mathrm{~mL}$ ) was added to the glass vial, and the solution was briefly degassed. The final reactants mol ratio was $[\mathrm{MA}] /[\mathrm{Asc}] /\left[\mathrm{CuBr}_{2}\right] /[\mathrm{PMDETA}] /[\mathrm{EBiBB}]=$ 8000:20:2:2:1. Consecutively, the monomer solution was transferred with a purged syringe to the flask containing the powders and was bubbled with nitrogen and thoroughly stirred for at least $1 \mathrm{~min} / \mathrm{mL}$ of solution. After that, the mixture containing the sacrificial initiator and the internal standard was added to the monomer solution to start the polymerization. Small volumes were subtracted at different reaction times and the reaction conversion and molecular weight estimates were analyzed by means of ${ }^{1} \mathrm{H}-\mathrm{NMR}$ measurements, using chloroform- $d_{6}$ as the deuterated solvent.

Characterization. Static contact angle measurements were performed on an optical contact angle device (OCA15, Dataphysics, Germany). Three water droplets were captured per sample, and the drop contour was fitted with the Young-Laplace equation. Results are shown in the Supporting Information (see Table S1 and Figure S1). Fourier transform infrared (FTIR) spectroscopy measurements were conducted on a Bruker $\alpha$ spectrometer for obtaining the characteristic group signals of the grafted PMA brushes. A bare silicon substrate was first used to generate a background spectrum. ${ }^{1} \mathrm{H}-\mathrm{NMR}$ was conducted on a Bruker Avance $400 \mathrm{MHz}$ instrument, using chloroform- $d_{6}$ as a solvent. AFM imaging of the polymer brush height was performed on a Multimode 8 AFM, using a NanoScope V controller and a JV vertical scanner. Tapping mode was the used operational mode, using aluminum-coated cantilevers, from Olympus. The selected cantilevers worked with a force constant of $2 \mathrm{~N} / \mathrm{m}$ and a resonance frequency of $\sim 70 \mathrm{kHz}$. X-ray photoelectron spectroscopy (XPS) was used for the determination of the chemical composition of the anchoring layers during the grafting density variation experiments. The measurements were carried out on a Quantera SXM (scanning XPS microprobe) from Physical Electronics, using a monochromatic $\mathrm{X}$-ray source $(\mathrm{Al} \mathrm{K \alpha}$, at $1486.6 \mathrm{eV})$. The obtained binding energy and spectra fitting was performed by shifting of the measured spectra with respect to known reference binding energies. In this case, aliphatic carbon $\mathrm{C} 1 \mathrm{~s}$ at $284.8 \mathrm{eV}$ was the selected reference.

Spectroscopic Ellipsometry Measurements. Measurements were performed on an M-2000X spectroscopic ellipsometer (J.A. Woollam) controlled by CompleteEASE software, operating in the wavelength range of $245-1000 \mathrm{~nm}$, with $5 \mathrm{~s}$ sampling time at three angles of incidence $\left(65,70\right.$, and $\left.75^{\circ}\right)$. For brush swelling measurements in acetone vapor, a $5 \mathrm{~mL}$ Heated Liquid Cell (J.A. Woollam) with windows fixed at an angle of incidence of $70^{\circ}$ was used. For liquid swelling measurements, acetone was injected directly into the cell using a syringe. Data analysis was performed using an optical model composed of the $\mathrm{Si}$ substrate, a $1 \mathrm{~nm}$ native $\mathrm{SiO}_{x}$ layer, and a Cauchy layer. A single Cauchy layer resulted in better fits than gradient layer models commonly employed for brushes in liquid. ${ }^{25-27}$ For the vaporsolvated brushes, this can be expected since neutron reflectivity measurements indicate block density profiles with a sharper decay than observed for liquid-solvated brushes. ${ }^{28}$ See the Supporting Information for further details on the ellipsometry models and experimental procedures (Figures S7, S8, and S9).

Quartz Crystal Microbalance Measurements. AT cut $10 \mathrm{MHz}$ quartz crystal sensors coated with a $50 \mathrm{~nm}$ layer of $\mathrm{SiO}_{2}$ (QSense QSQSX303) were functionalized with polymer brushes following the polymerization procedure, as described previously, with the exception that the sensors were plasma cleaned in $\mathrm{O}_{2}(0.3 \mathrm{mbar}, 50 \mathrm{~mA})$ for 4 min instead of piranha cleaning. The sensors were clamped in a 200 $\mu \mathrm{L}$ openQCM $\mathrm{Q}^{-1}$ cell connected to an HF2LI Lock-in Amplifier (Zurich Instruments) controlled via OpenLAB (Agilent). The cell was connected to a switch that could select a dry $\mathrm{N}_{2}$ line or a line containing an acetone bubbler $(330 \mathrm{~mL} / \mathrm{min})$. Before the measurements, the fundamental resonance frequency and third overtone were locked under dry $\mathrm{N}_{2}$. A typical measurement would start with $5 \mathrm{~min}$ of dry $\mathrm{N}_{2}, 5 \mathrm{~min}$ of acetone vapor, and this would be repeated once, finishing with $5 \mathrm{~min}$ of dry $\mathrm{N}_{2}$. To rule out pressure effects, a blank sample was also measured.

\section{RESULTS AND DISCUSSION}

Polymer Brush Preparation and Characterization. Scheme 1 shows the synthesis route that we employ to grow the PMA brushes. In short, first, $\mathrm{SiO}_{2}$ substrates are cleaned and activated in piranha solution. Next, the surface is functionalized with (3-aminopropyl)triethoxysilane (APTES) using vapor deposition (scheme 1a). Then, the $\alpha$-bromoisobutyryl bromide (BiBB) initiator molecules are coupled to the APTES-functionalized surface (Scheme $1 b_{1}$ ), and finally, the PMA brushes are grafted from the surface using SI-ARGETATRP (scheme 1c). Alternatively, we vary the grafting density by synthesizing brushes from APTES-functionalized surfaces that have a mixture of reactive $\mathrm{BiBB}$ initiators and nonreactive 1-bromocarbonyl-1-methylethyl acetate (BMA) molecules linked to them (scheme $1 b_{2}$ ). By varying the $[\mathrm{BiBB}] /[\mathrm{BMA}]$ molar ratio, we tune the grafting density. The change of active bromine species within the sample where polymer brushes would be able to bind was monitored by means of XPS. Three different samples were studied, containing: 100 vol $\%$ BiBB to 0 vol \% BMA, 50 vol \% BiBB to 50 vol \% BMA, and 0 vol \% $\mathrm{BiBB}$ to 100 vol \% BMA. Results show a decrease in the bromine atom signal at higher BMA ratios, as shown in Figure 1 and Table S2 in the Supporting Information. Samples with

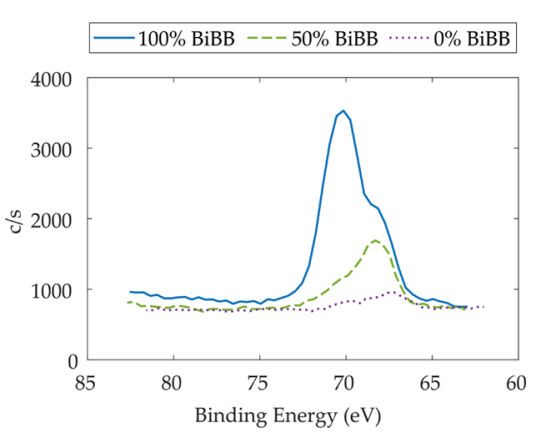

Figure 1. XPS spectra of bromine atom signal of silicon substrates functionalized with: $100 \% \mathrm{BiBB}$ (blue curve), $50 \% \mathrm{BiBB}$ (dashed green curve), and $0 \% \mathrm{BiBB}$ (dotted purple curve).

the highest active bromine moieties ( 0 vol \% inactive BMA) showed the strongest $\mathrm{Br}$ signal of $1.49 \%$, decreasing to $0.99 \%$ when increasing the concentration of inactive BMA to $50 \mathrm{vol}$ $\%$. Finally, when coating substrates only using a 100 vol \% inactive BMA solution, bromine atom traces were still found. Those are attributed to the reactions subproducts $(\mathrm{HBr})$ obtained on the binding of BMA molecules with the previously grafted anchoring layers. Interestingly, at $50 \mathrm{vol} \%$ of BIBB, the amount of active bromine species on the surface is higher than $50 \%$, even after correcting for byproducts. A reason for this is that at $50-50$ vol \% BIBB-BMA, the molar ratio is $1.18: 1$, such that there is slightly more BIBB in the solution. 
Moreover, BIBB is a smaller molecule than BMA and, therefore, more molecules can attach to the surface per unit area. Further details and XPS spectra can be found in the Supporting Information.

For the polymerization step, we developed a new synthesis route based on ref 29, where we utilize ascorbic acid (Asc) instead of $\mathrm{Cu}(0)$ as a reducing agent ${ }^{23}$ and growing the PMA macromolecules directly from initiators on the surface instead of in solution. This adaptation changes the polymerization kinetics. Thus, to achieve a controlled polymerization, we performed SI-ARGET-ATRP in dimethylformamide (DMF) using a monomer concentration of $5.5 \mathrm{M}$ and an optimized molar ratio of $[\mathrm{MA}] /[\mathrm{Asc}] /\left[\mathrm{CuBr}_{2}\right] /[\mathrm{PMDETA}]$ equal to 4000:10:1:1 (650 ppm vs monomer, with PMDETA being $N, N, N^{\prime}, N^{\prime \prime}, N^{\prime \prime}$-pentamethyldiethylenetriamine).

Figure $2 \mathrm{a}$ shows the dry height $h_{\mathrm{d}}$ of polymer brushes synthesized by the recipe described above, as measured by
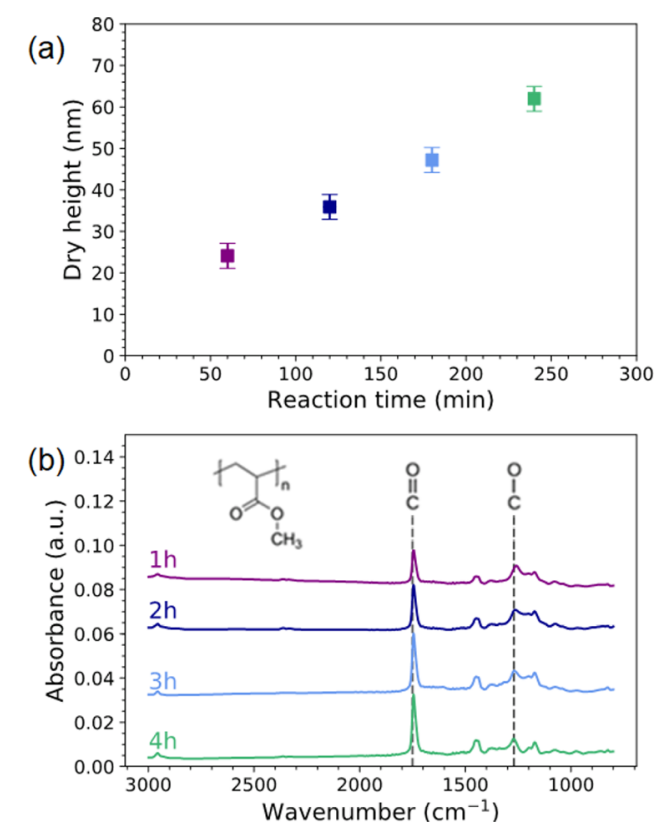

Figure 2. (a) Dry height of the PMA polymer brush as measured by $\mathrm{SE}$ as a function of reaction time. The error bars denote the standard error with a $95 \%$ confidence interval, but they are typically smaller than the symbol size. (b) Reflection mode FTIR-spectrum of the PMA brush grown on $\mathrm{SiO}_{2}$-coated QCM crystals for different polymerization times $(1-4 \mathrm{~h})$.

spectroscopic ellipsometry (SE) and fitted using the Cauchy model. Because PMA is hydrophobic, the height in air is not affected by the relative humidity. ${ }^{30}$ The linear increase in the height confirms good control of the polymerization. After a polymerization time of $1 \mathrm{~h}$, we obtain brushes with a dry height of approximately $22 \mathrm{~nm}$, and after $4 \mathrm{~h}$ of polymerization, the brush height is $65 \mathrm{~nm}$. Atomic force microscopy (AFM) measurements of the brush height corroborate the SE height measurements (see Figure S2 of the SI). From the dry height, we can extract the grafting density $\sigma$ of the brushes using

$$
\sigma=\frac{h_{\mathrm{d}} \rho N_{\mathrm{a}}}{M_{\mathrm{n}}}
$$

with $\rho$ being the PMA polymer density $\left(1.18 \mathrm{~g} / \mathrm{cm}^{3}\right), N_{\mathrm{a}}$ being Avogadro's number, and $M_{\mathrm{n}}$ being the number average molecular weight. We use ${ }^{1} \mathrm{H}-\mathrm{NMR}$ to determine the $M_{\mathrm{n}}$ of polymers grown in parallel in solution from sacrificial initiators (see Figure S5 of the SI). After a polymerization time of $4 \mathrm{~h}$, we find that $M_{\mathrm{n}}=242 \mathrm{kDa}$. By eq 1 , we calculate that the grafting density of our default brushes is $\sigma=0.10$ chains $/ \mathrm{nm}^{2}$. The difference in polymerization kinetics in solution and from the surface introduces an uncertainty in our grafting density (of typically $20-50 \%) .{ }^{19}$ Yet, in all cases, this means that we are in the high-density brush regime, ${ }^{31}$ as is commonly obtained by SI-ATRP. AFM imaging on our brushes of varied grafting densities (Figure S4 of the SI) confirms the presence of homogeneous brush films instead of low-density brushes, which would result in a rough and nonhomogeneous surface coverage, as shown in previous reports. ${ }^{32}$ The modified brushes with $\mathrm{BiBB} / \mathrm{BMA}$ combinations show grafting density values of $0.1,0.063,0.052$, and 0.042 chains $/ \mathrm{nm}^{2}$, with a decreasing concentration of $\mathrm{BiBB}$, respectively. Also here, we observe that the grafting density for 50-50 vol \% BIBB-BMA is larger than half the grafting density found for 100-0 vol \% BIBB-BMA. This is consistent with the bromine signal extracted from XPS results, which changed from $1.49 \%$ to only $0.99 \%$ when reducing the $\mathrm{BiBB}$ concentration from 100 to 50 vol \%. The difference of the brush dry height as a function of the grafting density is shown in Figure S3, and the AFM images confirm the morphology of the brush for high and low grafting densities in Figure S4 of the SI.

Fourier transform infrared (FTIR) measurements on brushes prepared for different polymerization times (see Figure $2 \mathrm{~b}$ ) confirm the success of the synthesis. The presence and the growth of the peaks at the vibrational modes of the acrylate carbonyl $(\mathrm{C}=\mathrm{O})$ at $1750 \mathrm{~cm}^{-1}$ and of the ester bond $(\mathrm{C}-\mathrm{O})$ at $1250 \mathrm{~cm}^{-1}$ are a strong indication that PMA polymers have been grafted from the surface.

Swelling of Polymer Brushes in Acetone: Liquid and Vapor States. Next, we examine the response of PMA brushes when exposed to acetone gas and liquid, respectively. The prepared PMA brushes swell strongly when brought in contact with acetone (see Figure 3a). The brushes (polymerized for $2 \mathrm{~h}$ ) have a height of $46 \mathrm{~nm}$ in a dry nitrogen atmosphere. When they are exposed to acetone vapor $(>5000 \mathrm{ppm})$, the brush height increases to $61 \mathrm{~nm}$, as measured by SE and fitted using the Cauchy model. We confirmed that the reduction in the refractive index $n$ is consistent with the effective medium approximation (EMA) ${ }^{33}$ within our experimental uncertainty: for Cauchy, we obtain $n=$ 1.460, and for EMA, we obtain $n=1.447$. The measured increase of $33 \%$ in the brush height is comparable to earlier reports for poly(methyl methacrylate) (PMMA) brushes, where the brush height was found to increase by $30 \%{ }^{34}$ This indicates that the solvation of these polymers is probably determined by the dipole interactions between the carbonyl group in acetone molecules and the ester moieties within the methacrylate side chains, which are identical for PMA and PMMA. Upon immersion of the brushes in liquid acetone, they swell by a factor 1.8 up to $82 \mathrm{~nm}$, which confirms that acetone is a good solvent for PMA. This is slightly smaller, but comparable to the swelling ratios of 1.9-2.0 observed for PMMA brushes of similar grafting densities. ${ }^{35}$

The swelling ratio of brushes immersed in liquid is known to increase upon decreasing the grafting density. ${ }^{35,36}$ Moreover, theoretically, a similar effect is predicted for brushes in mixtures of good and poor solvents (such as acetone vapor in air). ${ }^{37}$ Figure $3 \mathrm{~b}$ shows the swelling ratio of the brushes in liquid acetone as a function of the grafting density of the brush 

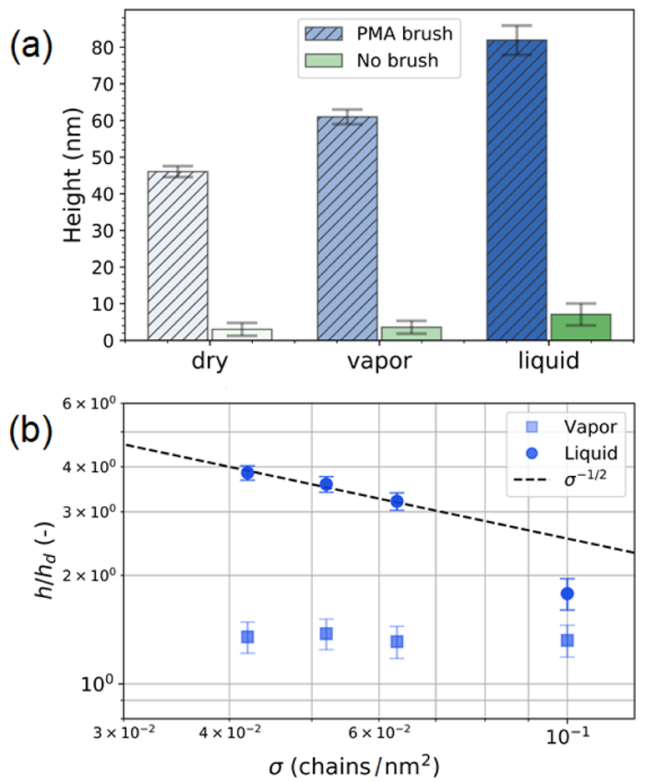

Figure 3. (a) Height of PMA brushes compared to APTESfunctionalized reference surfaces measured under dry nitrogen, acetone vapor (>5000 ppm), and in liquid acetone. (b) Swelling ratio $h / h_{\mathrm{d}}$ for PMA brushes of different grafting densities measured in liquid acetone (circles) and under acetone vapor (squares). The error bars depict the fit uncertainty of the ellipsometry data.

(dark blue circles). As expected, the swelling ratio in liquid acetone strongly increases upon decreasing the grafting density. For the lowest grafting density of 0.042 chains $/ \mathrm{nm}^{2}$, we find a swelling ratio of 3.85 . The swelling ratio decreases with a power-law scaling consistent with $h / h_{\mathrm{d}} \propto \sigma^{-1 / 2}$, as theoretically predicted. ${ }^{36}$ Only for the highest grafting density, we observe deviations. However, this is to be expected for very dense brushes as commonly obtained with SI-ATRP, ${ }^{35}$ because the theory presented in ref 36 assumes the brushes to be semidilute, which fails at high brush densities.

Surprisingly, the swelling ratio of brushes exposed to acetone vapor is independent of the grafting density at a value of 1.34 \pm 0.027 (see Figure $3 \mathrm{~b}$, light blue squares). The constant ellipsometry fitting parameters $(A$ and $B)$ and refractive index at $632 \mathrm{~nm}$ are also consistent with a constant swelling ratio, indicating that indeed the same solvent/polymer ratio is observed for all swelling ratios (see Figures S8 and S9 in the SI). Our results are in agreement with experimental observations by others, ${ }^{38}$ which show that the swelling ratio is independent of the grafting density for neutral hydrophilic brushes in contact with humid air. Yet, these results disagree with theoretical predictions based on a Flory Huggins ( $\mathrm{FH}$ ) type theory, which is derived for brushes by extending the $\mathrm{FH}$ free-energy equation with an entropic penalty for stretching of the end-grafted polymers. ${ }^{37}$ This penalty depends on the grafting density of the chains. Our observed insensitivity to the grafting density implies that the entropic penalty for stretching the end-anchored polymers is probably low compared to the other contributions to the FH free energy (mixing entropy and enthalpy). Thus, for our and similar noncharged systems, as discussed in ref 38, the traditional Flory Huggins theory for free polymer solvent mixtures, without explicitly incorporating end-anchoring of the polymers, ${ }^{34}$ appears to be sufficient to determine the chemical potential of solvent in brushes. As the traditional FH model is regularly employed in experimental studies, ${ }^{38-40}$ our results are an important confirmation of the validity of this. Additionally, the swelling ratio is independent of the length of the polymers in brush. For brushes with a dry height of $24 \mathrm{~nm}$, we observe a swelling ratio of 1.35 . Within the error margin, this is the same as the swelling ratio of the brushes with a dry height of $46 \mathrm{~nm}$. Therefore, our results imply that the response of PMA brushes to acetone vapor is robust, reproducible, and insensitive to variations in the grafting density, which is important for large-scale applications.

For sensing applications, also the response time is important. Figure $4 \mathrm{a}$ shows the change in the measured brush height as a
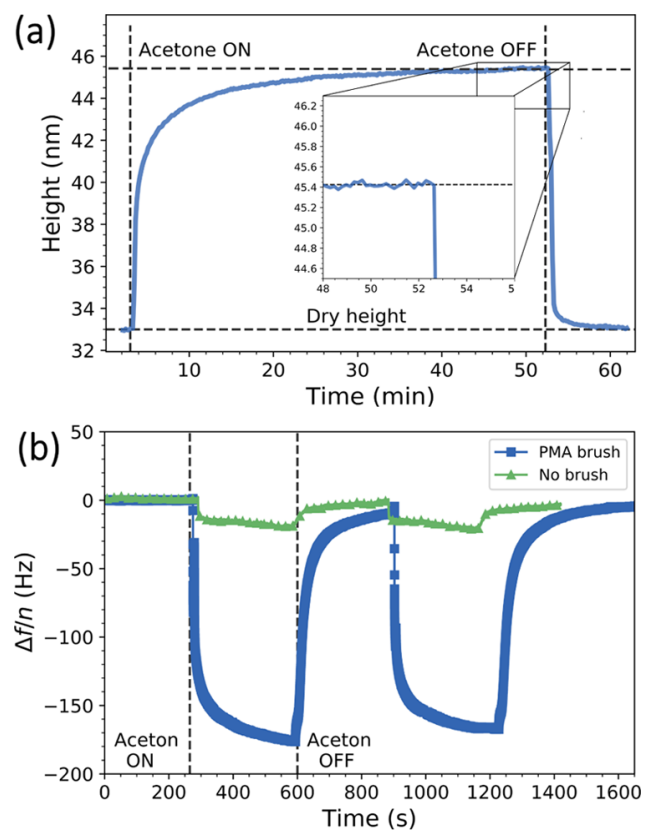

Figure 4. (a) Swelling response as measured by SE of the dense brush $\left(\sigma=0.1\right.$ chains $\left./ \mathrm{nm}^{2}\right)$ after exposure to acetone vapor at $t=0$ and to dry nitrogen gas at $t=53 \mathrm{~min}$ and (b) the measured change in oscillation frequency (fundamental) upon exposure to acetone vapor for a PMA brush versus a crystal with a brush as measured by QCM.

function of time after introducing the brush to acetone vapor (at $t=0$ ) and again to dry nitrogen at $t=53 \mathrm{~min}$ for a gas flow rate of $250 \mathrm{~mL} / \mathrm{min}$. Within a few seconds, the brush responds strongly to the acetone vapor and starts to swell and equilibrium is reached within $40 \mathrm{~min}$. This is much faster than the response time for spin-coated polymer films, for which it can take many hours to several days to reach equilibrium. ${ }^{41}$ The absorption time is independent of the grafting density (variations of around $5 \mathrm{~min}$ ). Instead, the absorption time for our brushes depends strongly on the flow rate: by doubling the flow rate, the response time decreases to approximately $25 \mathrm{~min}$ (see Figure $\mathrm{S} 10$ in the Supporting Information). This indicates that the response time is limited by the analyte supply and not by diffusion into the brush. This is consistent with the fast response times typically observed for brushes (on order of milliseconds) upon changing the solvent quality. ${ }^{42}$ Additionally, the time needed to dry the brush is much shorter than the time needed to solvate the brush, and after exposing the brush to dry nitrogen (at $t=53 \mathrm{~min}$ ), the dry height is reached within $5 \mathrm{~min}$. The reason for this might be the high vapor pressure of acetone as well as the higher flow rate of dry nitrogen, which both aid a fast evaporation of the absorbed liquid. 
Quartz Crystal Microbalance (QCM) Measurements. Gravimetric sensors, ${ }^{43}$ e.g., based on microcantilevers, ${ }^{16}$ are often employed in gas sensing. These sensors detect small changes in the mass adsorbed on the sensor surface, similar to quartz crystal microbalance (QCM) measurements. Therefore, we demonstrate the increased acetone sensing performance by incorporating PMA brushes on the sensor surface via QCM measurements. We determine the increase in sensitivity in mass sensing for QCM crystals coated with PMA brushes compared to reference substrates (APTES-functionalized $\mathrm{SiO}_{2}$ QCM crystals). Figure $4 \mathrm{~b}$ shows that the sensitivity $\left(\Delta f_{\text {brush }} /\right.$ $\left.\Delta f_{\text {nobrush }}\right)$ to a stepwise introduction to acetone vapor increases by at least a factor 6 in the first overtone with the addition of a PMA brush (see Figure S6 for data on the other overtones). This is a significant increase of the sensitivity and shows that the brushes can concentrate the analyte near the surface and, thereby, increase the sensitivity of sensors. Finally, we can calculate the thickness of the acetone film on the reference substrates. Since we know that the brush height increases by 15 $\mathrm{nm}$ when exposed to acetone vapor, we can estimate that a film of $15 \mathrm{~nm} / 6 \approx 2-3 \mathrm{~nm}$ adsorbs on APTES-covered $\mathrm{SiO}_{2}$. This value is consistent with the thickness increase of $1 \pm 2 \mathrm{~nm}$ we find in ellipsometry measurements. Moreover, this thickness is in agreement with estimates based on the Lifshitz theory for adsorption isotherms (see Figure S11 in the SI) for pressures relative to the saturation pressure between 0.88 and 0.97 , indicating that we performed our experiments close to the saturation pressure.

\section{CONCLUSIONS}

In summary, we have shown the successful synthesis of PMA brushes as well as their response to acetone exposure. We validate that the brushes swell strongly in liquid acetone and that the swelling ratio decreases for increasing grafting densities. When the brushes are brought in contact with acetone vapor, they swell by approximately $35 \%$. The brushes respond within seconds, and equilibrium is reached within 40 min. Interestingly, the swelling ratio for brushes in acetone vapor is independent of the grafting density, which makes them robust and reliable candidates for sensing applications. QCM measurements indicate that the sensitivity of mass sensors can be improved by a factor 6 using the PMA brush coatings. The next step will be to graft different brushes on sensor arrays, e.g., by inkjet printing, ${ }^{44}$ to explore possibilities for scent recognition via fingerprinting.

\section{ASSOCIATED CONTENT}

\section{SI Supporting Information}

The Supporting Information is available free of charge at https://pubs.acs.org/doi/10.1021/acs.langmuir.0c02510.

(1) Materials and methods, (2) supporting results, contact angle droplets (Figure S1); comparison SE and AFM (Figure S2); dry height of brushes as a function of grafting density (Figure S3); AFM imaging (Figure S4); H-NMR results (Figure S5); QCM frequency shifts (Figure S6); ellipsometry results (Figures S7, S8, and S9); flow rate influence on swelling experiments (Figure $\mathrm{S} 10)$; and (3) calculation acetone adsorption (PDF)

\section{AUTHOR INFORMATION}

\section{Corresponding Author}

Sissi de Beer - Materials Science and Technology of Polymers, University of Twente, 7522 NB Enschede, The Netherlands; (1) orcid.org/0000-0002-7208-6814; Phone: +31 (0)53 489 3170; Email: s.j.a.debeer@utwente.nl

\section{Authors}

Rens J. Horst - Materials Science and Technology of Polymers, University of Twente, 7522 NB Enschede, The Netherlands

Maria Brió Pérez - Materials Science and Technology of Polymers, University of Twente, 7522 NB Enschede, The Netherlands; orcid.org/0000-0002-6328-9556

Rick Cohen - Department of Chemistry, Saxion University of Applied Sciences, 7513 AB Enschede, The Netherlands

Marco Cirelli - Materials Science and Technology of Polymers, University of Twente, 7522 NB Enschede, The Netherlands; (1) orcid.org/0000-0001-7176-0566

Paloma S. Dueñas Robles - Materials Science and Technology of Polymers, University of Twente, 7522 NB Enschede, The Netherlands

Maria G. Elshof - Membrane Science and Technology Cluster, University of Twente, 7522 NB Enschede, The Netherlands

Aleksandar Andreski - Department of Nanotechnology, Saxion University of Applied Sciences, 7513 AB Enschede, The Netherlands

Mark A. Hempenius - Materials Science and Technology of Polymers, University of Twente, 7522 NB Enschede, The Netherlands

Nieck E. Benes - Membrane Science and Technology Cluster, University of Twente, 7522 NB Enschede, The Netherlands; (1) orcid.org/0000-0001-9716-069X

Cas Damen - Department of Nanotechnology, Saxion University of Applied Sciences, 7513 AB Enschede, The Netherlands

Complete contact information is available at: https://pubs.acs.org/10.1021/acs.langmuir.0c02510

\section{Author Contributions}

${ }^{\perp}$ R.J.H. and M.B.P. contributed equally

\section{Notes}

The authors declare no competing financial interest.

\section{ACKNOWLEDGMENTS}

The authors thank J. Paulusse for his help in interpreting the XPS data, C. Padberg for technical support, and G. Ebberink for support during the QCM measurements. This work is part of the research program "Mechanics of Moist Brushes" with project number OCENW.KLEIN.020, which is financed by the Dutch Research Council (NWO). In addition, the work has been financed by the Dutch Research Council (NWO), Holland Chemistry, the University of Twente, and the MESA+ Institute for Nanotechnology.

\section{REFERENCES}

(1) Wilson, A. D. Advances in Electronic-Nose Technologies for the Detection of Volatile Biomarker Metabolites in the Human Breath. Metabolites 2015, 5, 140-163.

(2) Loutfi, A.; Coradeschi, S.; Mani, G. K.; Shankar, P.; Rayappan, J. B. B. Electronic noses for food quality: A review. J. Food Eng. 2015, 144, 103-111.

(3) Yang, S.; Bintinger, J.; Park, S.; Jain, S.; Alexandrou, K.; Fruhmann, P.; Besar, K.; Katz, H.; Kymissis, I. Inexpensive, Versatile, 
and Robust USB-Driven Sensor Platform. IEEE Sens. Lett. 2017, 1, 14.

(4) Pelosi, P.; Zhu, J.; Knoll, W. From Gas Sensors to Biomimetic Artificial Noses. Chemosensors 2018, 6, No. 32.

(5) Firestein, S. How the olfactory system makes sense of scents. Nature 2001, 413, 211-218.

(6) Di Natale, C.; Paolesse, R.; Martinelli, E.; Capuano, R. Solidstate gas sensors for breath analysis: A review. Anal. Chim. Acta 2014, 824, 1-17.

(7) Borràs, E.; Ferré, J.; Boqué, R.; Mestres, M.; Acena, L.; Busto, O. Data fusion methodologies for food and beverage authentication and quality assessment: A review. Anal. Chim. Acta 2015, 891, 1-14.

(8) Chen, W.-L.; Cordero, R.; Tran, H.; Ober, C. K. 50th Anniversary Perspective: Polymer Brushes: Novel Surfaces for Future Materials. Macromolecules 2017, 50, 4089-4113.

(9) Chen, M.; Briscoe, W. H.; Armes, S. P.; Klein, J. Lubrication at Physiological Pressures by Polyzwitterionic Brushes. Science 2009, 323, 1698-1701.

(10) de Beer, S.; Kutnyanszky, E.; Schön, P. M.; Vancso, G. J.; Müser, M. H. Solvent induced immiscibility of polymer brushes eliminates dissipation channels. Nat. Commun. 2014, 5, No. 3781.

(11) Morgese, G.; Cavalli, E.; Rosenboom, J.-G.; Zenobi-Wong, M.; Benetti, E. M. Cyclic Polymer Grafts That Lubricate and Protect Damaged Cartilage. Angew. Chem., Int. Ed. 2018, 57, 1621-1626.

(12) Li, B.; Jain, P.; Ma, J.; Smith, J. K.; Yuan, Z.; Hung, H.-C.; He, Y.; Lin, X.; Wu, K.; Pfaendtner, J.; Jiang, S. Trimethylamine N-oxidederived zwitterionic polymers: A new class of ultralow fouling bioinspired materials. Sci. Adv. 2019, 5, No. eaaw9562.

(13) Christau, S.; Genzer, J.; von Klitzing, R. Polymer Brush/Metal Nanoparticle Hybrids for Optical Sensor Applications: from SelfAssembly to Tailored Functions and Nanoengineering. Z. Phys. Chem. 2014, 229, 1089-1117.

(14) Fortin, N.; Klok, H.-A. Glucose Monitoring Using a Polymer Brush Modified Polypropylene Hollow Fiber-based Hydraulic Flow Sensor. ACS Appl. Mater. Interfaces 2015, 7, 4631-4640.

(15) van Andel, E.; de Bus, I.; Tijhaar, E. J.; Smulders, M. M. J.; Savelkoul, H. F. J.; Zuilhof, H. Highly Specific Binding on Antifouling Zwitterionic Polymer-Coated Microbeads as Measured by Flow Cytometry. ACS Appl. Mater. Interfaces 2017, 9, 38211-38221.

(16) McCaig, H. C.; Myers, E.; Lewis, N. S.; Roukes, M. L. Vapor Sensing Characteristics of Nanoelectromechanical Chemical Sensors Functionalized Using Surface-Initiated Polymerization. Nano Lett. 2014, 14, 3728-3732.

(17) Righettoni, M.; Tricoli, A. Toward portable breath acetone analysis for diabetes detection. J. Breath Res. 2011, 5, No. 037109.

(18) Matyjaszewski, K.; Dong, H.; Jakubowski, W.; Pietrasik, J.; Kusumo, A. Grafting from Surfaces for Everyone: ARGET ATRP in the Presence of Air. Langmuir 2007, 23, 4528-4531.

(19) Zoppe, J. O.; Ataman, N. C.; Mocny, P.; Wang, J.; Moraes, J.; Klok, H.-A. Surface-Initiated Controlled Radical Polymerization: State-of-the-Art, Opportunities, and Challenges in Surface and Interface Engineering with Polymer Brushes. Chem. Rev. 2017, 117, $1105-1318$.

(20) Lemieux, M.; Usov, D.; Minko, S.; Stamm, M.; Shulha, H.; Tsukruk, V. V. Reorganization of binary polymer brushes: Reversible switching of surface microstructures and nanomechanical properties. Macromolecules 2003, 36, 7244-7255.

(21) Zhu, M.; Lerum, M. Z.; Chen, W. How to prepare reproducible, homogeneous, and hydrolytically stable aminosilanederived layers on silica. Langmuir 2012, 28, 416-423.

(22) Asenath Smith, E.; Chen, W. How to prevent the loss of surface functionality derived from aminosilanes. Langmuir 2008, 24, 1240512409 .

(23) Min, K.; Gao, H.; Matyjaszewski, K. Use of Ascorbic Acid as Reducing Agent for Synthesis of Well-Defined Polymers by ARGET ATRP. Macromolecules 2007, 40, 1789-1791.

(24) Matyjaszewski, K.; Dong, H.; Jakubowski, W.; Pietrasik, J.; Kusumo, A. Grafting from surfaces for "everyone": ARGET ATRP in the presence of air. Langmuir 2007, 23, 4528-4531.
(25) Kooij, E. S.; Sui, X.; Hempenius, M. A.; Zandvliet, H. J. W.; Vancso, G. J. Probing the Thermal Collapse of Poly(N-isopropylacrylamide) Grafts by Quantitative in Situ Ellipsometry. J. Phys. Chem. B 2012, 116, 9261-9268.

(26) Yu, Y.; Cirelli, M.; Kieviet, B. D.; Kooij, E. S.; Vancso, G. J.; de Beer, S. Tunable friction by employment of co-non-solvency of PNIPAM brushes. Polymer 2016, 102, 372-378.

(27) Ramakrishna, S. N.; Cirelli, M.; Divandari, M.; Benetti, E. M. Effects of Lateral Deformation by Thermoresponsive Polymer Brushes on the Measured Friction Forces. Langmuir 2017, 33, 4164-4171.

(28) Sun, L.; Akgun, B.; Hu, R.; Browning, J. F.; Wu, D. T.; Foster, M. D. Scaling Behavior and Segment Concentration Profile of Densely Grafted Polymer Brushes Swollen in Vapor. Langmuir 2016, $32,5623-5628$.

(29) Kwak, Y.; Magenau, A. J. D.; Matyjaszewski, K. ARGET ATRP of Methyl Acrylate with Inexpensive Ligands and ppm Concentrations of Catalyst. Macromolecules 2011, 44, 811-819.

(30) Ko, Y.; Miles, J. R.; Genzer, J. Determining Water Sorption and Desorption in Thin Hydrophilic Polymer Films by Thermal Treatment. ACS Appl. Polym. Mater. 2019, 1, 2495-2502.

(31) Patil, R. R.; Turgman-Cohen, S.; Srogl, J.; Kiserow, D.; Genzer, J. Direct Measurement of Molecular Weight and Grafting Density by Controlled and Quantitative Degrafting of Surface-Anchored Poly(methyl methacrylate). ACS Macro Lett. 2015, 4, 251-254.

(32) Benetti, E. M.; Zapotoczny, S.; Vancso, G. J. Tunable thermoresponsive polymeric platforms on gold by "photoiniferter"based surface grafting. Adv. Mater. 2007, 19, 268-271.

(33) Kappert, E. J.; Raaijmakers, M. J.; Tempelman, K.; Cuperus, F. P.; Ogieglo, W.; Benes, N. E. Swelling of 9 polymers commonly employed for solvent-resistant nanofiltration membranes: A comprehensive dataset. J. Membr. Sci. 2019, 569, 177-199.

(34) Orski, S. V.; Sheridan, R. J.; Chan, E. P.; Beers, K. L. Utilizing vapor swelling of surface-initiated polymer brushes to develop quantitative measurements of brush thermodynamics and grafting density. Polymer 2015, 72, 471-478.

(35) Moh, L. C. H.; Losego, M. D.; Braun, P. V. Solvent Quality Effects on Scaling Behavior of Poly(methyl methacrylate) Brushes in the Moderate- and High-Density Regimes. Langmuir 2011, 27, 36983702.

(36) Malham, I. B.; Bureau, L. Density Effects on Collapse, Compression, and Adhesion of Thermoresponsive Polymer Brushes. Langmuir 2010, 26, 4762-4768.

(37) Birshtein, T. M.; Lyatskaya, Y. V. Theory of the CollapseStretching Transition of a Polymer Brush in a Mixed Solvent. Macromolecules 1994, 27, 1256-1266.

(38) Galvin, C. J.; Genzer, J. Swelling of Hydrophilic Polymer Brushes by Water and Alcohol Vapors. Macromolecules 2016, 49, 4316-4329.

(39) Biesalski, M.; Rühe, J. Swelling of a Polyelectrolyte Brush in Humid Air. Langmuir 2000, 16, 1943-1950.

(40) Galvin, C. J.; Dimitriou, M. D.; Satija, S. K.; Genzer, J. Swelling of Polyelectrolyte and Polyzwitterion Brushes by Humid Vapors. J. Am. Chem. Soc. 2014, 136, 12737-12745.

(41) Koziara, B. T.; Akkilic, N.; Nijmeijer, K.; Benes, N. E. The effects of water on the morphology and the swelling behavior of sulfonated poly(ether ether ketone) films. J. Mater. Sci. 2016, 51, 1074-1082.

(42) Thomas, M.; Gajda, M.; Amiri Naini, C.; Franzka, S.; Ulbricht, M.; Hartmann, N. Poly(N,N-dimethylaminoethyl methacrylate) Brushes: pH-Dependent Switching Kinetics of a Surface-Grafted Thermoresponsive Polyelectrolyte. Langmuir 2015, 31, 1342613432.

(43) Fanget, S.; Hentz, S.; Puget, P.; Arcamone, J.; Matheron, M.; Colinet, E.; Andreucci, P.; Duraffourg, L.; Myers, E.; Roukes, M. Gas sensors based on gravimetric detection-A review. Sens. Actuators, $B$ 2011, 160, 804-821. 
(44) Kopeć, M.; Tas, S.; Cirelli, M.; van der Pol, R.; de Vries, I.; Vancso, G. J.; de Beer, S. Fluorescent Patterns by Selective Grafting of a Telechelic Polymer. ACS Appl. Polym. Mater. 2019, 1, 136-140. 